\title{
Hemorrhagic Pituitary Adenoma versus Rathke Cleft Cyst: A Frequent Dilemma
}

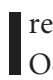
read with interest the article of Park et $\mathrm{al}^{1}$ published in the October issue of the American Journal of Neuroradiology. Differentiation with MR imaging of a cystic or hemorrhagic pituitary adenoma from a Rathke cleft cyst (RCC) remains a common issue. In daily practice, this situation may be particularly confusing in a young woman with mild hyperprolactinemia whose symptoms are frequently hidden by taking contraceptive pills. Moreover, parallelism between the prolactin level and tumoral volume is missing in hemorrhagic microprolactinomas. Then, diagnosis of hemorrhagic microprolactinoma versus T1 hyperintense intrasellar mucoid RCC is challenging.

Park et al reported that the main differentiating features of pituitary adenomas are off-midline location, tilting of the pituitary stalk, fluid-fluid level, T2 hypointense hemosiderin rim, and septations, while Rathke cleft cysts are more likely located on the midline and frequently present with a T2 hypointense characteristic nodule.

Nevertheless, pituitary adenomas may be on the midline, for instance corticotroph adenomas. The fluid-fluid level is inconstant, particularly with fresh hemorrhage and a peripheral hemosiderin rim because of the absence of blood-brain barrier in the pituitary gland; septations are inconstant. On the other hand, Rathke cleft cysts may be, rarely, in an off-midline location, and their T2 hypointense waxy nodules are detected in no more than $70 \%$ of cases.

Moreover, the diagnostic tree model proposed by Park et al seems difficult to apply to the strictly intrasellar infracentimetric

http://dx.doi.org/10.3174/ajnr.A4653 lesions, which are more and more frequently seen with highresolution $3 \mathrm{~T}$ scanners, either discovered fortuitously or in the assessment of hyperprolactinemia.

Ancillary signs are then welcome to differentiate RCCs from cystic or hemorrhagic pituitary adenomas.

The axial T1-weighted sequence is optimal for making the diagnosis of such RCCs: Strict midline location, regular convex symmetric anterior surface, and close contact with the posterior lobe are characteristic features. ${ }^{2}$

Furthermore, while intrasellar pituitary adenomas, even tiny ones, give rise to mass effect, such as bulging of the sellar diaphragm and eroding of the bony contours of the sella, intrasellar RCCs of equal volume give rise to a less important mass effect or even to no mass effect, most probably because of intracystic low pressure, at least with an asymptomatic RCC (Fig 1).

This observation is helpful in the presence of the not-so-rare concomitant intrasellar pituitary adenoma and RCC, in which the soft RCC is deformed by the firmer pituitary adenoma, even if the latter is smaller (Fig 2).

\section{REFERENCES}

1. Park M, Lee SK, Choi J, et al. Differentiation between cystic pituitary adenomas and Rathke cleft cysts: a diagnostic model using MRI. AJNR Am J Neuroradiol 2015;36:1866-73 CrossRef Medline

2. Bonneville JF, Bonneville F, Cattin F. Magnetic resonance imaging of pituitary adenomas. Eur Radiol 2005;15:543-48 CrossRef Medline

(D).-F. Bonneville

Department of Radiology and Endocrinology University Hospital of Liège, University of Liège

Liège, Belgium 

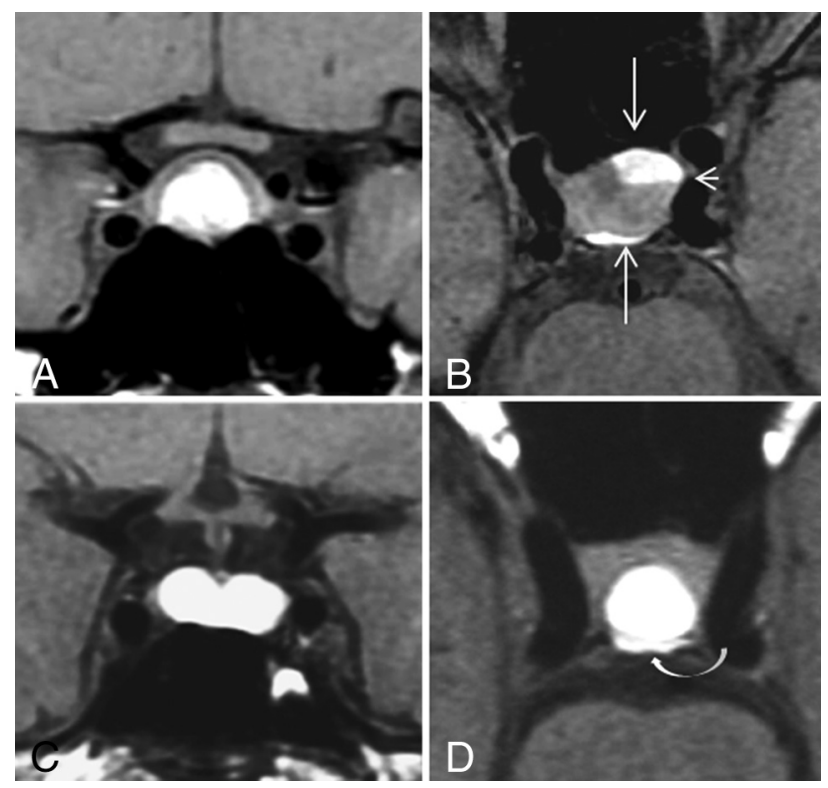

FIG 1. Hemorrhagic pituitary adenoma ( $A$ and $B$ ) versus mucoid RCC $(C$ and $D)$ on coronal and axial TTWI. This hemorrhagic pituitary adenoma ( $A$ and $B$ ) has fluid-fluid levels (short arrow) and mass effect on the sellar diaphragm, posterior lobe, and anterior wall of the sella (long arrows). In this RCC ( $C$ and $D$ ), note a mild imprint on the posterior lobe but no deformation of the sellar diaphragm or of the anterior wall.

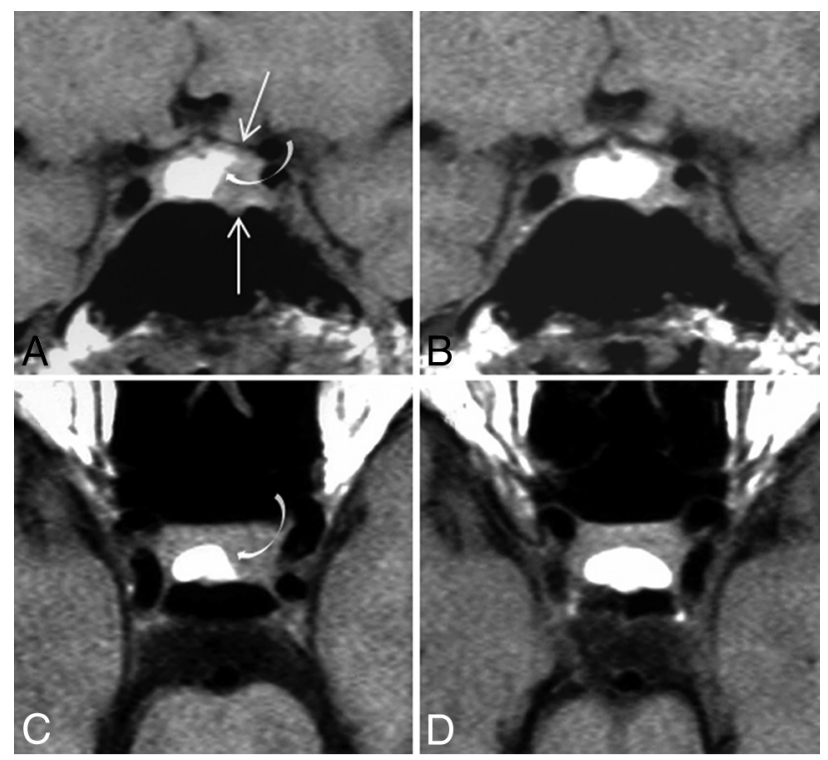

FIG 2. Concomitant pituitary adenoma and RCC. $A$ and $B$, Coronal TIWI before and after cabergoline treatment. $C$ and $D$, Corresponding axial TTWI. The $T 1$ isointense pituitary microadenoma deforms the sellar floor, upraises the upper surface of the gland (long arrows), and imprints the lateral margin of a TT hyperintense mucoid RCC (curved arrow). After treatment ( $B$ and $D$ ), shrinkage of the adenoma leads to a re-expansion of the RCC, whose pattern is pathognomonic in axial TIWI. D, Note again the absence of mass effect. 